\title{
English Language Teacher Education from the Lens of Critical Pedagogy
}

\author{
Kamile Hamiloğlu
}

PhD, Marmara University

\section{Abstract}

In English (as a foreign) language teacher education, there have been numerous theories which have been applied, adapted, and reconstructed based on the needs and conditions of the learners and teachers from very early times so far, that is, from the appearance of Behaviourism in foreign language education in the 1940s to the present time. There have always been many transitions and shifts among and within these theories explaining the practices in English (as foreign language) classes. This paper examines how these theories and transitions can be considered from the lens of Critical Pedagogy and it interrogates whether the recognition of the Critical Pedagogy may help foreign language teacher education turn to be transformative and whether it may contribute to the development of the conditions for a better world within and outside the school with the help of teachers trained to be critical. The theoretical framework of this chapter is built on the socio-cultural view, socio-cognitive view, transformative view and Critical Pedagogy in foreign language education. However, earlier theories such as the Behaviouristic Approach, the Cognitive Approach, and the Communicative Approach are visited as background knowledge. Given that the sociocultural approach portrays human learning as a social process and the origination of human intelligence in society or culture referring to the fact that social interaction plays a basic role in the development of cognition, the socio-cognitive view deals with how language is learned and should be treated; and transformative learning gives way to actual behaviour that learners should establish to contribute to and participate in the community where they live in; these theories in the framework shed light on developing understandings of the recognition of Critical Pedagogy in the foreign language teacher education. Regarding this overview, it is seen that socio-cultural and socio-cognitive transformation could be led by critical pedagogy, in schools initially, then in the whole society, addressing radical concerns and the abuses of power in intercultural contexts in the acquisition of foreign languages and in their circulation by the help of critically trained foreign language teachers.

Keywords: transformative foreign language education, critical pedagogy, critical reflection, EFL, EFL materials 